\title{
EVALUACIÓN DE SUPERVIVENCIA Y DESARROLLO INICIAL DE 42 PROGENIES DE PEUMO (Cryptocarya alba (Mol.) Looser) ESTABLECIDAS EN LA PROVINCIA DE ARAUCO, REGIÓN DEL BIOBÍO, CHILE
}

\author{
Gutiérrez, Braulio $^{13}$ y Chung, PatricioPo ${ }^{13}$
}

\section{RESUMEN}

Se analiza la supervivencia y crecimiento inicial de un ensayo de 42 progenies y 18 procedencias de peumo (Cryptocarya alba) de 15 meses de edad, establecido en la comuna de Cañete (Provincia de Arauco, región del Biobío).

Se evalúa también el efecto de dos tipos de protectores individuales de plantas sobre la supervivencia y crecimiento del ensayo.

El ensayo presenta alta supervivencia, pero un crecimiento muy modesto, observándose diferencias significativas de estas variables entre progenies (entre y dentro de las procedencias).

Se observa también un positivo efecto de los protectores de malla raschell en supervivencia y crecimiento en altura de la plantas.

Palabras clave: Cryptocarya alba, progenie, procedencia, crecimiento inicial, supervivencia, protectores individuales de plantas

\section{SUMMARY}

Survival and early growth of a 42 progenies and 18 provenances trial of peumo (Cryptocarya alba), established in the commune of Cañete (Province of Arauco, Biobío) was analyzed at 15 months old.

The effect of two kinds of individual tree shelters on survival and early growth of the trial was also evaluated.

The trial has high survival but a very modest growth, showing significant differences in these variables between progenies (between and within provenances).

A positive effect of raschell-net tree shelters over survival and height growth of the plants was observed.

Keywords: Cryptocarya alba, progeny, provenance, early growth, survival, tree shelters

${ }^{13}$ Ingeniero Forestal. Instituto Forestal, Sede Biobío. bgutierr@infor.cl; pchung@infor.cl 


\section{INTRODUCCIÓN}

El peumo es un árbol nativo que crece desde el sur de la provincia del Limarí hasta la provincia de Cautín, siendo más abundante en la zona central de Chile que al sur de su distribución (Martin, 1989). Se le puede encontrar en la ladera occidental de la Cordillera de la Costa, el Valle Central y el sector andino, a altitudes que pueden llegar a los 1.500 msnm (Rodríguez et al., 1983).

En su amplia distribución presenta una significativa variación que no ha sido cuantificada, pero que resulta evidente al comparar, por ejemplo, las características morfológicas de frutos colectados desde distintas procedencias (Chung, 2015).

La especie posee numerosos usos tradicionales que se aprovechan fundamentalmente en el mundo rural. Sus frutos son comestibles (Vogel et al., 2008; Barreau y Salas, 2009; Chung, 2012) y tienen aplicación en gastronomía (Boragó, 2011 cit. por Chung, 2015); en medicina tradicional las infusiones de hojas y de corteza se usan para enfermedades hepáticas (Montes, 1987) y en hemorragias y reumatismos (Vogel et al., 2008), existiendo diversas otras aplicaciones medicinales señaladas por distintos autores (Ibaca, 2001; Barreau y Salas, 2009); y el alto contenido de taninos y pigmentos de su corteza permite usarlo en el procesamiento de cuero y en la tintura de lana (Sapaj, 1998; Vogel et al., 2008).

Peumos es además una especie muy adecuada para proteger las laderas de los cursos de agua y para la forestación de sectores húmedos y sombríos (Hoffmann, 1983); sus bosques son grandes productores de hojarasca, que se usa como tierra de hoja (Sapaj, 1998); se la utiliza también como ornamental, gracias a su follaje denso, siempreverde, brillante y de llamativos frutos rojos (Hoffmann, 1983); además se usa para leña y carbón, y como forraje para los animales (Martín, 1989; cit. por Vogel et al., 2008).

Independiente de los usos anteriores, la especie concentra un creciente interés debido a atributos químicos de sus taninos, alcaloides y aceites esenciales. Diversos estudios han demostrado que componentes químicos extraídos desde especies de Cryptocarya exhiben actividad contra el cáncer, mientras que otros poseen efectos antibióticos de distinta naturaleza (Chung, 2015).

Para el género se han descrito del orden de 40 alcaloides, la mayoría de los cuales poseen propiedades antitumorales, bactericidas, antimicrobianos, fungicidas, insecticidas 0 antioxidantes (Toribio et al., 2006). La reticulina, un alcaloide extraído del peumo, posee propiedades hepatoprotectoras (Montes y Wilkomirsky, 1985).

Por su parte, estudios efectuados con los aceites esenciales de las hojas de Cryptocarya alba, identifican 71 compuestos orgánicos volátiles (Karimi et al., 2011), algunos de los cuales muestran actividad contra el protozoo causante del mal de Chagas, contra Leishmania spp $^{14}$ (Schmeda-Hirschmann et al., 2001) y contra Staphylococcus aureus (Avello et al., 2012).

Según el Libro Rojo de la Flora Terrestre de Chile, el peumo se encuentra en la categoría de vulnerable en la Precordillera de Santiago (CONAF, 1989; Niemeyer et al., 2002). Arancio et al. (2001), la incluyen en esta misma categoría (vulnerable) señalando que posee problemas de conservación en la región de Coquimbo.

En general las amenazas que enfrenta la especie provienen de la sobreexplotación y destrucción de su hábitat. Estos factores no solo producen una reducción del tamaño de la población y del flujo de genes, sino que también pueden provocar la eliminación total de las poblaciones locales; además, la fragmentación de sus bosques puede conducir al aislamiento genético de una población continua, lo que a su vez causaría más pérdida en la diversidad genética como resultado de la endogamia y la deriva genética (De Moraes et al., 2002).

\footnotetext{
${ }^{14}$ Agente causante de enfermedades que van desde úlceras cutáneas, hasta inflamaciones graves del hígado y bazo que pueden resultar fatales.
} 
La diversidad de usos tradicionales de la especie, y fundamentalmente las aplicaciones potenciales de sus características químicas, junto con la variabilidad exhibida y las amenazas que enfrenta su conservación, motivaron al Instituto Forestal a estudiar la variabilidad de peumo e iniciar actividades tendientes a su conservación y mejoramiento genético, con el fin de aprovechar las interesantes propiedades de la especie y particularmente de sus frutos.

La instalación de ensayos genéticos, donde se representen en un sitio común a progenies de árboles selectos, permitirá generar importante información que contribuya a una selección más apropiada de los individuos a considerar para la producción de frutos de interés.

En este ámbito, en agosto de 2014 se estableció un primer ensayo de progenies de peumo, respecto del cual este documento tiene por objetivo presentar sus resultados de supervivencia y desarrollo inicial, a nivel de progenies y procedencia del material ensayado.

\section{MATERIAL Y METODO}

\section{Ensayo}

El ensayo evaluado fue establecido en agosto de 2014, en terrenos del Museo Mapuche de Cañete, en la provincia de Arauco, región del Biobío (UTM 18H 641305.36 - 581276.30; 80 msnm).

El ensayo consiste de 25 bloques, en cada uno de los cuales se representan 42 progenies de peumo a razón de una planta de cada progenie en cada bloque.

Las madres de cada progenie corresponden a árboles de peumo seleccionados en distintas localidades de la región del Biobío, en función de la abundancia en su producción de frutos (Cuadro $N^{\circ} 1$ ).

En su plantación se utilizaron plantas de una temporada de viverización, producidas en speedling trays de 84 cavidades de $130 \mathrm{~cm}^{3}$ cada una, usando como sustrato corteza compostada de pino, enriquecida con un fertilizante comercial de liberación controlada, con relación NPK de 16:8:12 a razón de $3 \mathrm{~kg} / \mathrm{m}^{3}$ de sustrato.

Durante la viverización las plantas fueron manejadas de acuerdo al programa operacional del vivero institucional de INFOR Biobío.

La plantación del ensayo se realizó a un espaciamiento de $2,5 \times 2,5 \mathrm{~m}$ en casillas efectuadas sobre un terreno previamente rozado y tratado con herbicida de preplantación.

Inmediatamente después de la plantación se fertilizó las plantas con producto de lenta entrega y relación NPK 15:8:15, a razón de $50 \mathrm{~g} /$ planta.

Posteriormente se rodeó cada planta con un protector individual; en 12 bloques se uso malla rashell de $80 \%$ de sombreamiento y una longitud de $80 \mathrm{~cm}$, sostenida con tres estacas de madera alrededor de la planta; en los otros 13 bloques se usó secciones de $30 \mathrm{~cm}$ de una manga continua de malla plástica semirrígida, de $10 \mathrm{~cm}$ de diámetro y sostenida alrededor de la planta mediante una varilla de alambre (Figura $\mathrm{N}^{\circ} 1$ ). 
Cuadro $\mathrm{N}^{\circ} 1$

UBICACIÓN DE LOS ÁRBOLES MADRES DE LAS PROGENIES CONTEMPLADAS EN EL ENSAYO

\begin{tabular}{|c|c|c|c|c|c|c|}
\hline \multirow{2}{*}{ Progenie } & \multirow{2}{*}{ Procedencia } & \multicolumn{3}{|c|}{ Coordenadas UTM } & \multirow{2}{*}{$\begin{array}{c}\text { Altitud } \\
\text { (msnm) }\end{array}$} & \multirow{2}{*}{ Ubicación } \\
\hline & & Uso & Lat & Long & & \\
\hline 1 & Copiulemu & $18 \mathrm{H}$ & 693697 & 5918613 & 227 & Secano Interior \\
\hline 2 & Copiulemu & $18 \mathrm{H}$ & 693798 & 5917209 & 151 & Secano Interior \\
\hline 3 & Tucapel & $19 \mathrm{H}$ & 237785 & 5867491 & 321 & Precordillera \\
\hline 4 & Tucapel & $19 \mathrm{H}$ & 237771 & 5867495 & 319 & Precordillera \\
\hline 5 & Cerro Yanqui & $19 \mathrm{H}$ & 249223 & 5862593 & 417 & Precordillera \\
\hline 6 & Antuco & $19 \mathrm{H}$ & 265408 & 5865366 & 560 & Precordillera \\
\hline 7 & Yumbel & $18 \mathrm{H}$ & 716935 & 5902156 & 103 & Valle Central \\
\hline 8 & Yumbel & $18 \mathrm{H}$ & 716935 & 5902156 & 103 & Valle Central \\
\hline 9 & Yumbel & $18 \mathrm{H}$ & 716935 & 5902156 & 103 & Valle Central \\
\hline 10 & Yumbel & $18 \mathrm{H}$ & 716935 & 5902156 & 103 & Valle Central \\
\hline 11 & El Rosal & $18 \mathrm{H}$ & 733500 & 5875168 & 138 & Valle Central \\
\hline 12 & El Rosal & $18 \mathrm{H}$ & 733500 & 5875168 & 138 & Valle Central \\
\hline 13 & El Rosal & $18 \mathrm{H}$ & 733500 & 5875168 & 138 & Valle Central \\
\hline 14 & Quilleco & $19 \mathrm{H}$ & 236477 & 5848778 & 332 & Precordillera \\
\hline 15 & Polcura & $19 \mathrm{H}$ & 259150 & 5870195 & 536 & Precordillera \\
\hline 16 & Santa Juana & $18 \mathrm{H}$ & 679806 & 5903005 & 48 & Secano Costero \\
\hline 17 & Santa Juana & $18 \mathrm{H}$ & 679632 & 5902873 & 41 & Secano Costero \\
\hline 18 & Nacimiento & $18 \mathrm{H}$ & 680851 & 5874834 & 155 & Secano Interior \\
\hline 19 & Nacimiento & $18 \mathrm{H}$ & 680851 & 5874834 & 155 & Secano Interior \\
\hline 20 & Nacimiento & $18 \mathrm{H}$ & 680851 & 5874834 & 155 & Secano Interior \\
\hline 21 & Nacimiento & $18 \mathrm{H}$ & 695194 & 5856264 & 134 & Secano Interior \\
\hline 22 & Carrizal - Los Patos & $18 \mathrm{H}$ & 689901 & 5851327 & 236 & Secano Interior \\
\hline 23 & Carrizal - Los Patos & $18 \mathrm{H}$ & 687257 & 5851689 & 286 & Secano Interior \\
\hline 24 & Carrizal - Los Patos & $18 \mathrm{H}$ & 684406 & 5851780 & 326 & Secano Interior \\
\hline 25 & Culenco & $18 \mathrm{H}$ & 750701 & 5900164 & 168 & Valle Central \\
\hline 26 & Culenco & $18 \mathrm{H}$ & 750793 & 5900403 & 173 & Valle Central \\
\hline 27 & Culenco & $18 \mathrm{H}$ & 750792 & 5900492 & 160 & Valle Central \\
\hline 28 & Curapaso & $18 \mathrm{H}$ & 757990 & 5890695 & 214 & Valle Central \\
\hline 29 & La Obra & $18 \mathrm{H}$ & 757316 & 5891624 & 193 & Valle Central \\
\hline 30 & La Obra & $18 \mathrm{H}$ & 757316 & 5891624 & 203 & Valle Central \\
\hline 31 & La Obra & $18 \mathrm{H}$ & 757316 & 5891624 & 208 & Valle Central \\
\hline 32 & Quillaimavida & $18 \mathrm{H}$ & 698913 & 5911292 & 301 & Secano Interior \\
\hline 33 & Quillaimavida & $18 \mathrm{H}$ & 699117 & 5910962 & 300 & Secano Interior \\
\hline 34 & Quillaimavida & $18 \mathrm{H}$ & 699115 & 5910967 & 298 & Secano Interior \\
\hline 35 & Quillaimavida & $18 \mathrm{H}$ & 699091 & 5911013 & 297 & Secano Interior \\
\hline 36 & Quillaimavida & $18 \mathrm{H}$ & 699100 & 5911001 & 295 & Secano Interior \\
\hline 37 & Quillaimavida & $18 \mathrm{H}$ & 699227 & 5910616 & 286 & Secano Interior \\
\hline 38 & Tomeco & $18 \mathrm{H}$ & 703441 & 5906928 & 252 & Secano Interior \\
\hline 39 & Tomeco & $18 \mathrm{H}$ & 703441 & 5906928 & 254 & Secano Interior \\
\hline 40 & Quillón & $18 \mathrm{H}$ & 726090 & 5930727 & 67 & Valle Central \\
\hline 41 & Coronel & $18 \mathrm{H}$ & 666483 & 5920256 & 10 & Secano Costero \\
\hline 42 & Coronel & $18 \mathrm{H}$ & 666483 & 5920256 & 10 & Secano Costero \\
\hline
\end{tabular}




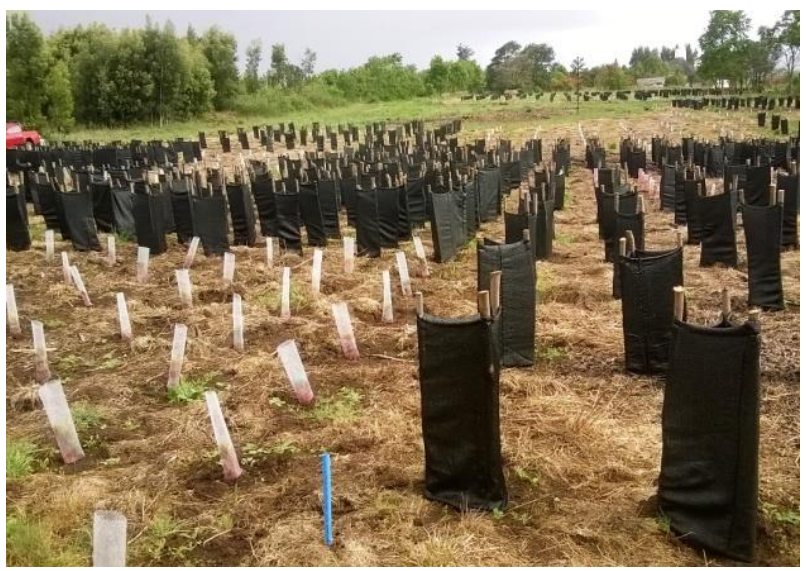

Figura $\mathrm{N}^{\circ} 1$

DETALLE DE LOS DOS TIPOS DE MALLA UTILIZADAS COMO PROTECTORES INDIVIDUALES PARA LAS PLANTAS DEL ENSAYO DE PROGENIES DE PEUMO

\section{Evaluación}

En octubre de 2015, 15 meses después de su establecimiento, se midió la altura (H) y diámetro de cuello (D) de todas las plantas que componen el ensayo, y se contabilizó la supervivencia (S) de las mismas.

Para evaluar el efecto de los dos tipos de malla usados como protectores, se analizó el ensayo como un modelo completamente aleatorizado, considerando a los bloques de progenies como repeticiones de cada uno de los dos tratamientos evaluados (protector de malla rashell repetido 12 veces; protector de malla semirrígida repetido 13 veces). Se contabilizó la supervivencia en cada tratamiento y mediante análisis de varianza se determinó el efecto de estos tratamientos sobre las variables de crecimiento evaluadas (altura y diámetro de cuello).

A nivel de procedencias y progenies se realizó un análisis estadístico descriptivo para las variables altura, diámetro y supervivencia. Este se complementó con análisis de varianza y pruebas de comparación múltiple de medias (Prueba de Scott y Knott; alfa=0,05), con la finalidad de detectar diferencias estadísticamente significativas a nivel de procedencia y progenies. En esta evaluación se usó un modelo de bloques completos al azar, donde las procedencias y progenies fueron los tratamientos evaluados en 25 bloques. De este modo, la variación se segregó entre los tratamientos (procedencias y progenies), separando la fracción de la variación debida al efecto de los protectores, la que quedó enmascarada en la fuente de variación correspondiente a los bloques.

\section{RESULTADOS Y DISCUSIÓN}

\section{Situación General del Ensayo y Efecto de Mallas de Protección}

A los 15 meses de edad el ensayo de progenies de peumo presenta una supervivencia de $94,3 \%$, y sus plantas exhiben una altura media de $10,7 \mathrm{~cm}$ y un diámetro de cuello de $2,3 \mathrm{~mm}$. Se observan diferencias estadísticamente significativas entre las plantas que se establecieron con diferente tipo de protector; las plantas establecidas con malla rashell presentan mayores valores de altura y supervivencia y son además más homogéneas que las plantas protegidas con malla semirrígida. Estas últimas, además de tener un desempeño inferior son más heterogéneas, exhibiendo una desviación estándar mayor, así como valores máximos y mínimos más extremos que en el primer caso. Respecto al diámetro de cuello, este es poco variable en el ensayo y no se 
detectan diferencias en función del tipo de protector, sin embargo persiste la tendencia a que las plantas protegidas con malla semirrígida presenten mayor variabilidad, en términos de valores máximos mínimos, que aquellas protegidas con malla rashell (Cuadro $\mathrm{N}^{\circ} 2$ )

Cuadro $\mathrm{N}^{\circ} 2$

VARIABLES DESCRIPTIVAS DEL ENSAYO Y DIFERENCIAS SEGÚN TIPO DE PROTECTOR

\begin{tabular}{|c|c|c|c|c|}
\hline \multirow{2}{*}{\multicolumn{2}{|c|}{ Variable }} & \multicolumn{2}{|c|}{ Tipo de protector } & \multirow{3}{*}{$\begin{array}{r}\text { Total } \\
94,3 \\
\end{array}$} \\
\hline & & \multirow{2}{*}{$\begin{array}{c}\begin{array}{c}\text { Malla } \\
\text { Raschell }\end{array} \\
98,0\end{array}$} & \multirow{2}{*}{$\begin{array}{r}\text { Malla } \\
\text { Semirrígida } \\
90,8\end{array}$} & \\
\hline \multirow{4}{*}{ Supervivencia (\%) } & Prom & & & \\
\hline & D.E. & 4,0 & 9,0 & 5,5 \\
\hline & Max & 100,0 & 100,0 & 100,0 \\
\hline & Min & 83,3 & 61,5 & 76,0 \\
\hline \multirow{4}{*}{ Altura (m) } & Prom & 12,2 & 9,3 & 10,7 \\
\hline & D.E. & 4,1 & 5,4 & 5,0 \\
\hline & Max & 30,1 & 50,0 & 50,0 \\
\hline & Min & 3,0 & 2,0 & 2,0 \\
\hline \multirow{4}{*}{ Diámetro de Cuello $(\mathrm{mm})$} & Prom & 2,3 & 2,3 & 2,3 \\
\hline & D.E. & 0,6 & 0,6 & 0,6 \\
\hline & Max & 4,2 & 4,4 & 4,4 \\
\hline & Min & 0,9 & 0.5 & 0,5 \\
\hline
\end{tabular}

El uso de protectores individuales para las plantas puede mejorar la supervivencia y el crecimiento inicial de las mismas, brindándole condiciones microambientales más favorables y protegiéndolas del daño provocado por animales. En el caso de este ensayo, la malla raschell efectivamente genera un ambiente distinto alrededor de la planta (menor insolación, evaporación, temperatura, mayor humedad menor desecación por viento, etc.), lo que influye en el crecimiento y supervivencia de las mismas. En cambio, la malla semirrígida, con una trama muy rala no tiene mayor efecto que el de brindar protección mecánica contra conejos o liebres. En la práctica, la situación resulta similar a la de comparar plantas con protector (malla raschell) versus plantas sin protector (malla semirrígida).

Generalmente las plantas que cuentan con protectores exhiben mayor crecimiento inicial en altura que aquellas que carecen de esta protección. En efecto, diversos estudios indican que en los primeros estadios de desarrollo, los protectores producen plantas con tallos más largos y menos ramificados, pero de menor diámetro que el de plantas sin protector. La respuesta a la sombra y la reducción de la radiación, junto con el menor efecto del viento y de estrés mecánico, permite que las plantas con protector concentren más energía en el crecimiento en altura, y no así en el crecimiento diamétrico o radicular (Jacobson y Jackson, s/f).

Los resultados observados en el ensayo analizado coinciden con la tendencia mencionada en la bibliografía. La comparación de medias mediante la prueba de Tuckey (alfa= $0,05)$ no detecta diferencias significativas en diámetro, pero si para la altura y supervivencia de las plantas con protector (malla raschell) respecto a las protegidas con malla semirrígida. Estas últimas, que en la práctica no cuentan con un microambiente modificado por el protector, exhiben un $31 \%$ menos de altura que las protegidas con malla raschell $(9,3 \mathrm{vs} 12,2 \mathrm{~cm})$ y casi un $8 \%$ menos de supervivencia. Los valores, aunque significativos, son menores a los reportados por Jacobson y Jackson (s/f) para otros ensayos, donde la diferencia de altura entre las plantas con y sin protectores, durante su desarrollo inicial, puede llegar casi al 100\% (las protegidas casi duplican a las sin protector); o incluso exceder del 100\%, como indica Randall (2012) para plantas de Quercus spp, las que después de un año de establecidas en terreno, registraron diferencias de altura de $121 \%$ a favor de plantas protegidas respecto de los testigos sin protector (31 vs $14 \mathrm{~cm}$, respectivamente). 


\section{Crecimiento y Supervivencia por Procedencias y Progenies}

Los resultados de los análisis de varianza para altura y diámetro de cuello indican la existencia de diferencias estadísticamente significativas entre progenies, tanto entre como dentro de las procedencias (Cuadro $\mathrm{N}^{\circ} 3$ ).

A nivel de procedencias se pueden individualizar tres grupos de altura, donde destacan con los mejores valores aquellas correspondientes a Coronel, Quillaimavida, Tucapel, Culenco y Santa Juana, mientras que los peores desempeños son los de las procedencias Curapaso, Quilleco, Polcura y Antuco; las procedencias restantes conforman un grupo de altura intermedia entre los dos anteriores.

En términos de diámetro de cuello se observa una situación similar, definiéndose tres grupos que con algunas variaciones son similares a los de altura. La correlación entre ambas variables es alta a nivel de procedencias $\left(r_{H-D C}=0,82\right)$, de modo que en general los grupos de mayor altura son también los de mejor diámetro de cuello.

Como procedencia individual destaca Coronel que alcanza los mayores valores en ambas variables; Santa Juana con altos valores de altura y desempeño intermedio en diámetro es la única procedencia que registra $100 \%$ de supervivencia; por su parte Polcura y Antuco exhiben simultáneamente los valores más bajos en supervivencia, altura y diámetro de cuello.

La correlación entre supervivencia y variables de crecimiento es intermedia $\left(r_{S-H}=0,64\right.$ y $r_{S-D C}=0,52$ ), no observándose tendencias evidentes que relacionen a estas variables. Tampoco se observa una relación clara entre el comportamiento de las procedencias y su ubicación fisiográfica, aunque se detecta cierta tendencia a que las procedencias de precordillera exhiban un comportamiento más deficitario que las restantes, tanto en crecimiento como en supervivencia; complementariamente, las procedencias de secano, particularmente de secano costero, tienden a presentar mejor desempeño que las procedencias de los orígenes restantes.

A nivel de progenies, en general se observa un comportamiento coherente con el de las procedencias a que pertenecen, es decir que las procedencias de buen desempeño tienen progenies de buen desempeño y viceversa.

No obstante, también se detecta variación entre las progenies dentro de una misma procedencia. Este es el caso, por ejemplo, del material de Culenco, donde dos de sus progenies (progenies 26 y 27) se encuentran entre las de mejor comportamiento en altura, diámetro y supervivencia, mientras que la tercera (progenie 25) exhibe uno de los peores resultados en las tres variables.

En términos de medias por progenies, se distinguen dos grupos estadísticamente homogéneos en altura y cuatro en diámetro, observándose al igual que en el nivel de procedencias, una correlación relativamente alta entre estas dos variables $\left(r_{H-D C}=0,79\right)$. Análogamente también, las correlaciones entre supervivencia y variables de crecimiento son menores, e incluso más bajas que las determinadas a nivel de procedencias $\left(r_{S-H}=0,52\right.$ y $\left.r_{S-D C}=0,46\right)$.

Las mejores progenies (8, 42, 26 y 27) pertenecen a Yumbel, Coronel y Culenco, mientras que las de menor crecimiento y supervivencia $(25,15$ y 6$)$ corresponden a Culenco, Polcura y Antuco. Los valores específicos de cada variable a nivel de progenies y procedencias se detallan en el Cuadro $\mathrm{N}^{\circ} 3$. 
Cuadro $\mathrm{N}^{\circ} 3$

MEDIAS DE SUPERVIVENCIA, ALTURA Y DIÁMETRO DE CUELLO POR PROCEDENCIAS Y PROGENIES DE ENSAYO DE PEUMO EVALUADO A 15 MESES DE EDAD

\begin{tabular}{|c|c|c|c|c|c|c|c|c|c|c|c|c|c|}
\hline Procedencia & $\mathbf{n}$ & $\mathrm{s}$ & $\mathrm{H}$ & & DC & & Prog & $\mathbf{n}$ & S & H & & DC & \\
\hline \multirow{2}{*}{ Coronel } & \multirow{2}{*}{45} & \multirow{2}{*}{90,0} & \multirow{2}{*}{12,2} & \multirow{2}{*}{$a$} & \multirow{2}{*}{2,5} & \multirow{2}{*}{$a$} & 42 & 23 & 92,0 & 13,1 & $a$ & 2,5 & $a$ \\
\hline & & & & & & & 41 & 22 & 88,0 & 11,4 & $\mathbf{a}$ & 2,5 & $\mathrm{~b}$ \\
\hline \multirow{6}{*}{ Quillaimavida } & \multirow{6}{*}{146} & \multirow{6}{*}{97,3} & \multirow{6}{*}{11,9} & \multirow{6}{*}{$a$} & \multirow{6}{*}{2,4} & \multirow{6}{*}{$a$} & 32 & 25 & 100,0 & 12,8 & $a$ & 2,9 & $a$ \\
\hline & & & & & & & 34 & 25 & 100,0 & 12,6 & $\mathbf{a}$ & 2,3 & $b$ \\
\hline & & & & & & & 36 & 25 & 100,0 & 12,1 & $a$ & 2,7 & $a$ \\
\hline & & & & & & & 35 & 24 & 96,0 & 11,7 & \begin{tabular}{|l}
$a$ \\
\end{tabular} & 2,4 & $\mathrm{a}$ \\
\hline & & & & & & & 37 & 22 & 88,0 & 11,4 & $a$ & 1,9 & $\mathrm{~d}$ \\
\hline & & & & & & & 33 & 25 & 100,0 & 11,1 & $\mathbf{a}$ & 2,1 & C \\
\hline \multirow{2}{*}{ Tucapel } & \multirow{2}{*}{47} & \multirow{2}{*}{94,0} & \multirow{2}{*}{11,8} & \multirow{2}{*}{$a$} & \multirow{2}{*}{2,4} & 0 & 4 & 24 & 96,0 & 12,2 & $\mathbf{a}$ & 2,4 & $\mathrm{~b}$ \\
\hline & & & & & & a & 3 & 23 & 92,0 & 11,5 & $\mathbf{a}$ & 2,4 & $\mathrm{~b}$ \\
\hline & & & & & & & 26 & 25 & 100,0 & 12,9 & $\mathbf{a}$ & 2,7 & $a$ \\
\hline Culenco & 70 & 93,3 & 11,5 & a & 2,4 & $a$ & 27 & 25 & 100,0 & 12,9 & $a$ & 2,7 & a \\
\hline & & & & & & & 25 & 20 & 80,0 & 8,2 & b & 1,8 & $\mathrm{~d}$ \\
\hline & & & & & & & 17 & 25 & 100,0 & 11,7 & $\mathbf{a}$ & 2,3 & $\mathrm{~b}$ \\
\hline Santa Juana & 50 & 100,0 & 11,5 & a & 2,1 & b & 16 & 25 & 100,0 & 11,3 & $a$ & 1,9 & $\mathrm{~d}$ \\
\hline Coniulemи & & & & & & & 1 & 24 & 96,0 & 10,8 & $\mathbf{a}$ & 2,4 & $\mathrm{~b}$ \\
\hline Copıulemu & 49 & 98,0 & 10,8 & b & 2,3 & $a$ & 2 & 25 & 100,0 & 10,8 & $a$ & 2,3 & $\mathrm{~b}$ \\
\hline & & & & & & & 24 & 24 & 96,0 & 12,2 & $a$ & 2,5 & $a$ \\
\hline Carrizal Los & 72 & 96,0 & 10,7 & b & 2,2 & $a$ & 22 & 25 & 100,0 & 11,1 & $\mathbf{a}$ & 2,2 & $b$ \\
\hline & & & & & & & 23 & 23 & 92,0 & 8,7 & b & 1,9 & $\mathrm{~d}$ \\
\hline & & & & & & & 18 & 24 & 96,0 & 12,8 & $\mathbf{a}$ & 2,4 & $\mathrm{~b}$ \\
\hline & & & & & & & 21 & 22 & 88,0 & 12,2 & $a$ & 2,3 & $\mathrm{~b}$ \\
\hline Nacimiento & 94 & 94,0 & 10,7 & b & 2,1 & $b$ & 19 & 24 & 96,0 & 9,1 & b & 2,0 & $d$ \\
\hline & & & & & & & 20 & 24 & 96,0 & 8,7 & b & 1,8 & $d$ \\
\hline & & & & & & & 8 & 23 & 92,0 & 13,3 & $\mathbf{a}$ & 2,7 & $\mathrm{a}$ \\
\hline & & & & & & & 9 & 22 & 88,0 & 9,7 & b & 2,4 & $\mathrm{~b}$ \\
\hline Yumbel & 91 & 91,0 & 10,5 & b & 2,3 & $\mathrm{a}$ & 7 & 24 & 96,0 & 9,7 & b & 2,1 & C \\
\hline & & & & & & & 10 & 22 & 88,0 & 9,3 & b & 2,1 & $\mathrm{C}$ \\
\hline Tomern & 47 & 940 & 104 & $\mathbf{h}$ & 22 & $a$ & 38 & 24 & 96,0 & 11,9 & $\mathbf{a}$ & 2,6 & $a$ \\
\hline eco & 47 & 94,0 & 10,4 & $\mathbf{D}$ & 2,2 & a & 39 & 23 & 92,0 & 9,0 & b & 1,9 & $d$ \\
\hline & & & & & & & 11 & 23 & 92,0 & 11,1 & $\mathbf{a}$ & 2,3 & $\mathrm{~b}$ \\
\hline El Rosal & 70 & 93,3 & 10,3 & b & 2,1 & $b$ & 12 & 23 & 92,0 & 10,3 & b & 2,1 & C \\
\hline & & & & & & & 13 & 24 & 96,0 & 9,6 & b & 2,1 & C \\
\hline & & & & & & & 31 & 25 & 100,0 & 11,6 & $a$ & 2,3 & $\mathrm{~b}$ \\
\hline La Obra & 74 & 98,7 & 10,3 & b & 2.3 & $a$ & 30 & 25 & 100,0 & 10,8 & $a$ & 2.8 & $a$ \\
\hline & & & & & & & 29 & 24 & 96,0 & 8,3 & b & 1,9 & $d$ \\
\hline Cerro Yanqui & 24 & 96,0 & 10,0 & b & 2,2 & $a$ & 5 & 24 & 96,0 & 10,0 & b & 2,2 & C \\
\hline Quillón & 24 & 96,0 & 9,6 & b & 2,3 & $\mathrm{a}$ & 40 & 24 & 96,0 & 9,6 & b & 2,3 & $\mathrm{~b}$ \\
\hline Curapaso & 23 & 92,0 & 8,6 & C & 2,0 & $\mathrm{~b}$ & 28 & 23 & 92,0 & 8,6 & b & 2,0 & $d$ \\
\hline Quilleco & 23 & 92,0 & 8,3 & C & 2,1 & $\mathrm{~b}$ & 14 & 23 & 92,0 & 8,3 & b & 2,1 & $\mathrm{C}$ \\
\hline Polcura & 22 & 88,0 & 8,0 & C & 1,7 & C & 15 & 22 & 88,0 & 8,0 & b & 1,7 & $d$ \\
\hline Antuco & 19 & 76,0 & 7,0 & C & 1,8 & C & 6 & 19 & 76,0 & 7,0 & b & 1,8 & $d$ \\
\hline Total & 990 & 94,3 & 10,7 & & 2,3 & & & 990 & 94,3 & 10,7 & & 2,3 & \\
\hline
\end{tabular}

Letras distintas en una misma columna indican diferencias estadísticamente significativas entre medias

(Scott y Knott, alfa=0,05)

Las procedencias y progenies evaluadas en el ensayo presentan una supervivencia satisfactoria, con un valor medio de $94,3 \%$, valores extremos familiares de 100 y $76 \%$, y con solo 8 de 42 progenies bajo el 90\%. Al respecto, Quiroz et al. (2012) en plantaciones de restauración 
efectuadas con esta especie, también señalan altas tasas de supervivencia incluso sin uso de protectores. Por el contrario, plantaciones de un año de edad establecidas en Los Vilos y Rapel logran una supervivencia menor; $72 \%$ en la primera localidad y 10 a $37 \%$ en la segunda (Barros y Schickhardt, 1978; 1979).

Contrariamente el crecimiento resulta deficitario. Tras 15 meses en terreno las plantas presentan altura media de $10,7 \mathrm{~cm}$ y un diámetro de cuello de $2,3 \mathrm{~mm}$, valores que no representan un incremento significativo respecto del que tenían las plantas al final de su viverización, antes de ser plantadas en el ensayo (INFOR, 2014). Esta diferencia se explica en gran medida por el hecho de que muchas plantas sufrieron la desecación de la parte aérea durante su primer periodo estival en terreno, de modo que al momento de la medición solo exhibían un pequeño rebrote basal de escasa altura y diámetro. En efecto, plantaciones efectuadas en condiciones más adversas y con considerable menor supervivencia, como las mencionadas por Barros y Schickhardt $(1978 ; 1979)$, exhibían después de un año en terreno valores medios de altura que fluctuaban entre 12,6 y 16,1 $\mathrm{cm}$, los que resultan mayores a los observados en este ensayo.

\section{CONCLUSIONES}

Los protectores individuales de malla raschell exhibieron un positivo efecto sobre la supervivencia y crecimiento en altura de las plantas de peumo.

Existen diferencias de crecimiento y supervivencia entre las progenies ensayadas, estas diferencias ocurren entre y dentro de las procedencias, observándose cierta tendencia hacia un mejor desempeño de las procedencias de secano costero y un rendimiento más deficitario de las de precordillera.

La supervivencia es alta y satisfactoria, no así el crecimiento inicial, que es escaso como consecuencia de desecación de plantas.

Para efectos de selección de material genético la evaluación resulta demasiado incipiente, se debe mantener el ensayo y repetir la evaluación en estadios de desarrollo más avanzados.

\section{REFERENCIAS}

Arancio, G.; Muñoz, M. y Squeo, F., 2001. Descripción de Algunas Especies con Problemas de Conservación en la IV Región de Coquimbo, Chile. En: Libro Rojo de la Flora Nativa y de los Sitios Prioritarios para su Conservación: Región de Coquimbo. F.A. Squeo, G. Arancio y J.R. Gutiérrez, Eds. Ediciones Universidad de La Serena, La Serena, Chile 6: 63 - 103

Avello, M.; López, C.; Gatica, C.; Bustos, E.; Brieva, A.; Pastene, E. y Bittner, M., 2012. Efectos antimicrobianos de extractos de plantas chilenas de las familias Lauraceae y Atherospermataceae. Revista Cubana de Plantas Medicinales. 2012; 17(1)73-83.

Barreau, A. y Salas, V., 2009. Plantas que curan, compartiendo vivencias y saberes. Medicina campesina de la Región del Biobío. TAC- Forestal Mininco. 73 p.

Barros, S. y Schickhardt, R., 1978. Resultados de prendimiento y desarrollo de 22 especies, en zonas áridas sometidas a diferentes métodos de plantación. Los Vilos IV región. Instituto Forestal. Informe Técnico $N^{\circ} 70$. Santiago. $24 \mathrm{p}$.

Barros, S. y Schickhardt, R., 1979. Ensayos de métodos de plantación e introducción de especies en zonas áridas y semiáridas. Rapel. Instituto Forestal. Informe Técnico N62. Santiago. 24 p.

Chung, P., 2012. Usos, Productos y Procesos. En: Benedetti, S. (Ed.). Monografía de Peumo, Cryptocarya alba (Mol.) Looser. INFOR - MINAGRI. Programa de investigación de productos forestales no madereros. Pp: 57-58.

Chung, P., 2015. Iniciativas de conservación en especies nativas: El caso del peumo. En: Gutiérrez, B.; Ipinza, R.; y Barros, S. (Eds). Conservación de recursos genéticos forestales: Principios y prácticas. Instituto Forestal. 320 p. Capítulo 14, Pp. 249-268. 
Corporación Nacional Forestal (CONAF) 1989. Libro Rojo de la Flora Terrestres de Chile. BENOIT, I (Ed.). Ministerio de Agricultura, Santiago, $157 \mathrm{pp}$.

De Moraes, P.; Monteiro, R. y Vencovsky, R., 2002. Genetic differentiation and diversity of natural populations of Cryptocarya spp. (Lauraceae) from the Brazilian Atlantic rain forest. Lundiana 3(2): 99-109.

Hoffmann, A., 1983. El árbol urbano de Chile. Santiago, Chile. Ediciones Fundación Claudio Gay. 225 p.

Ibaca, R., 2001. Monografía de árboles y arbustos chilenos con propiedades medicinales aromáticas. Memorias para optar al título de Ingeniero Forestal. Universidad de Concepción, Facultad de Ciencias Forestales, Departamento de Silvicultura. $246 \mathrm{p}$.

INFOR, 2014. Producción de plantas e instalación de ensayo de progenies de peumo (Cryptocarya alba (Mol.) Looser). Informe Interno Grupo Conservación y Mejoramiento Genético. INFOR Sede Biobío. Concepción. 24 p.

Jacobson, M. and Jackson, D., s/f. Tree Shelters: A Multipurpose Forest Management Tool. Penn. State College of Agricultural Sciences. Forest Finance Series Publications $N^{\circ}$ 7. En: http://extension.psu.edu/naturalresources/forests/finance/forest-tax-info/publications/tree-shelters.

Karimi, H.; Farmani, A. y Nourizadeh, H., 2011. A performance comparison of modern statistical technique for molecular descriptor selection and retention prediction of essential oil from leaves. American Journal of Scientific Research. 38: 36-40.

Martin, F., 1989. Extraíbles químicos de especies nativas en zonas áridas y semiáridas. Revisión Bibliográfica. Documento de Trabajo № 24. Proyecto CONAF/PNUD/FAO-CHI/83/017. 43 p.

Montes, M., 1987. Aspectos de la medicación Popular en la Región del Biobío, Chile. Acta Farm. Bonaerense. $6(2): 115-24$.

Montes, M. y Wilkomirsky, T., 1985. Medicina tradicional chilena. Concepción, Chile. Universidad de Concepción. 206 p.

Niemeyer, H.; Bustamante, R.; Simonetti, N.; Teillier, S.; Fuentes, E. y Mella, J., 2002. Historia Natural de la Reserva Nacional Río Clarillo: un espacio para aprender ecología. Tercer Curso Regional de Actualización en Biología de la Conservación. Santiago, Chile. 315 p.

Quiroz, I.; Pincheira, M. y Hernández, A., 2012. Restauración Ecológica en la Zona Central de Chile: Técnicas silvícolas para la supervivencia y crecimiento de especies nativas bajo condiciones de estrés hídrico. Informe interno Centro Tecnológico de la Planta Forestal. INFOR.

Randall, J., 2012. Tree shelter for hardwood plantings. F-364. lowa State University, Forestry Extension. En: https://www.extension.iastate.edu/forestry/publications/PDF_files/F-364.pdf

Rodríguez, R.; Matthei, O. y Quezada M., 1983. Flora arbórea de Chile. Concepción, Chile. Editorial de la Universidad de Concepción. 408 p.

Schmeda-Hirschmann, G.; Astudillo, L.; Bastida, J.; Codina, C.; De Arias, A; Ferreira, M; Inchaustti, A. and Yaluff, G., 2001. Cryptofolione derivatives from Cryptocarya alba fruits. Journal of Pharmacy and Pharmacology, 53: 563-567.

Sapaj, A., 1998. Potencialidad del bosque esclerófilo del Valle de Colliguay (V Región) para la obtención de productos secundarios. Memorias para optar al título profesional de Ingeniero Forestal. Universidad de Chile, Facultad de Ciencias Forestales, Escuela de Ciencias Forestales, Departamento de Silvicultura. 108 p.

Toribio, A.; Bonfils, A.; Delannay, E.; Prost, E.; Harakat, D.; Henon, E.; Richard, B.; Litaudon, M.; Nuzillard, J. y Renault, J., 2006. Novel seco-Dibenzopyrrocoline Alkaloid from Cryptocarya oubatchensis. Organic Letters. 8(17): $3825-3828$

Vogel, H.; Razmilic, I.; San Martín, J.; Doll, U. y González, B., 2008. Plantas Medicinales Chilenas. Experiencia de Domesticación y Cultivo de Boldo, Matico, Bailahuén, Canelo, Peumo y Maqui. Segunda Edición. Editorial de la Universidad de Talca. $194 \mathrm{p}$. 